American Journal of Applied Sciences 7 (9): 1215-1218, 2010

ISSN 1546-9239

(C) 2010 Science Publications

\title{
Effect of Thickness of Tris (8-Hydroxyquinolinato) Aluminum on the Photoluminescence and I-V Characteristic of Organic Light Emitting Structure
}

\author{
${ }^{1}$ Lim Mei Yee, ${ }^{1}$ Wan Mahmood Mat Yunus, \\ ${ }^{1}$ Zainal Abidin Talib and ${ }^{2}$ Anuar Kassim \\ ${ }^{1}$ Department of Physics, \\ ${ }^{2}$ Department of Chemistry, \\ Faculty of Science, University Putra Malaysia, \\ 43400 UPM Serdang, Selangor, Malaysia
}

\begin{abstract}
Problem statement: How the thickness of the tris (8-hydroxyquinolinato) Aluminum $\left(\mathrm{Alq}_{3}\right)$ effect the optical and electrical properties of organic light emitting diode. Approach: The optimum thickness, photoluminescence and current-voltage characteristic of $\mathrm{Alq}_{3}$ layer on N, N-bis (inaphthyl)$\mathrm{N}, \mathrm{N}$-diphenyl-1,1-biphenyl-4,4-diamine $(55 \mathrm{~nm}$ ) layer in Organic Light-Emitting Devices (OLED) structure are reported. $\mathrm{Alq}_{3}$ and NPB organic layers are used as Electron Transport Layer (ETL) and as Hole Transport Layer (HTL) in Organic Light-Emitting Devices (OLED). The thin layers of the NPB and $\mathrm{Alq}_{3}$ were prepared by thermal evaporation method. Results: The $\mathrm{Alq}_{3}$ layer was evaporated on the NPB layer for thickness ranging from 16 to $134 \mathrm{~nm}$ and photoluminescence and I-V characteristic were studied using fiber optics spectrophotometer (Ocean Optics- USB 2000 FLG) and current-voltage source (Keithley, model 2400). Conclusion: It was found that the $\mathrm{Alq}_{3}$ with $84 \mathrm{~nm}$ thicknesses gives the highest photoluminescence peak at $520 \mathrm{~nm}$ wavelengths, as well as the lowest turn on voltage of the device. The optical reflectance spectra for every sample were also reported.
\end{abstract}

Key words: Photoluminescence, hydroxyquinolinato, electrical conductivity, optical reflectance

\section{INTRODUCTION}

Currently, there is great research has been demonstrated in the study of Organic Light-Emitting Devices (OLEDs) for full-color display application (Lim et al., 2010a; 2010b). OLEDs have an organic EL medium consisting of extremely thin layers sandwiched by two electrodes. In a basic two-layer OLEDs structure, one organic layer is specifically chosen to transport holes and the other organic layer is to transport electrons. When an electrical potential difference is applied between the anode and the cathode such that the anode is at a more positive electrical potential with respect to the cathode, injection of holes occurs from the anode into the Hole Transport Layer (HTL), while electrons are injected from the cathode into the Electron Transport Layer (ETL) (Zhao et al., 2006; Coe et al., 2002; Kanno et al., 2004; Yoon et al., 2007).

The advantage of Organic LEDs compared with inorganic LEDs is their ease of manufactured, high efficiency of emission light with the low operating voltage. There are severally organic material that can be used as HTL such as N, N`-bis (Inaphthyl)-N, N`diphenyl-1, 1'-biphenyl-4, 4'-diamine (NPB) and N, N'-diphenyl-N, N'-bis (3-methylphenyl) (1, 1'biphenyl)-4, 4'-Diamine (TPD). NPB is the most widely used as a HTL. NPB can be easily manufactured and is abundantly available in powder form. However, Tris (8-hydroxyquinolinato) Aluminum $\left(\mathrm{Alq}_{3}\right)$ used as a ETL because $\mathrm{Alq}_{3}$ is thermally and morphologically stable to be evaporated into thin films form, easily synthesized and purified, molecularly shaped to avoid exciplex formation (e.g., with N, N'-bis (Inaphthyl)-N,N'-diphenyl-1,1'biphenyl-4,4'-diamine at the interface) and with green fluorescent it become a good host emitter (Hung and Chen, 2002). Thus, optimum thickness of the $\mathrm{Alq}_{3}$ is very important to improve the performance of the OLEDs. Therefore in this study we report the effect of thickness of $\mathrm{Alq}_{3}$ on the electrical conductivity, turn on voltage and photoluminescence of the $\mathrm{Alq}_{3}$ as hole transport layer in OLED device.

Corresponding Author: Wan Mahmood Mat Yunus, Department of Physics, Faculty of Science, University Putra Malaysia, 43400 UPM Serdang, Selangor, Malaysia Tel: + (603)89466684 Fax: + (603)89454454 


\section{MATERIALS AND METHODS}

In this experiment, we used $\mathrm{Alq}_{3}$ as the ETL and $\mathrm{N}$, N'- bis (inaphthyl) - N, N'-diphenyl-1, 1'-biphenyl-4, 4'-diamine (NPB) as the HTL, Indium Tin Oxide (ITO) as the anode Aluminum (Al) as a cathode, respectively. Devices with a structure of ITO/NPB $(55 \mathrm{~nm}) / \mathrm{lq}_{3}(\mathrm{~T}$ $\mathrm{nm}) / \mathrm{Al}(300 \mathrm{~nm})$, where $\mathrm{T}$ was chosen as $16,33,50$, 67, 84, 101, 118 and $134 \mathrm{~nm}$, were fabricated (Fig. 2). NPB and $\mathrm{Alq}_{3}$ compounds were purchased from SigmaAldrich. The organic materials were deposited by thermal evaporation onto Indium Tin Oxide (ITO) glass substrates with a sheet resistance of about $15 \Omega$ at room temperature in a high vacuum chamber (Fig. 1). The ITO glass was immersed in ultrasonic bath with acetone for $10 \mathrm{~min}$. Then, the ITO glass was rinsed in deionized water for $10 \mathrm{~min}$ and then blow dry with nitrogen gas. This procedure was used to remove organic contamination and particles from the ITO surface.

Current-voltage measurement was carried out using current-voltage source (Keithley, model 2400). The thickness of the layers was measured by disk profile machine (Tencor P-12). The Photoluminescence (PL) of the films was measured using Spectrofluorometer (Oceon Optics 2000 FLG). Spectrophotometer and Micro Autolab Type III Potentiostat were used to for measuring energy band gap and cyclic voltametry study.

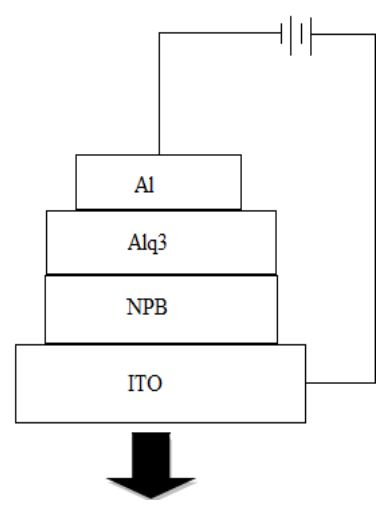

Fig. 1: The structure of OLED device

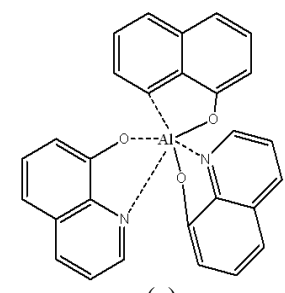

(a)

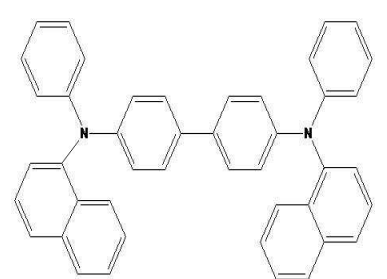

(b)
Fig. 2: Molecular structure of (a) tris (8hydroxyquinolinato) aluminum $\left(\mathrm{Alq}_{3}\right)$ and (b) $\mathrm{N}, \quad$ N'-bis(Inaphthyl)-N, N'-diphenyl-1,1'biphenyl-4,4'-diamine, (MPB)

\section{RESULTS}

Photoluminescence is the reemission of light after absorbing a photon of higher energy (Bouzid et al., 2005). Figure 3 shows the photoluminescence spectrum for devices with various thickness of $\mathrm{Alq}_{3}$.

The reflection spectra in Fig. 4 and 5 were taken during an OLED process in which double layers were stacked on the substrates: A Hole Transport Layer (HTL) and an Electron Transport Layer (ETL).

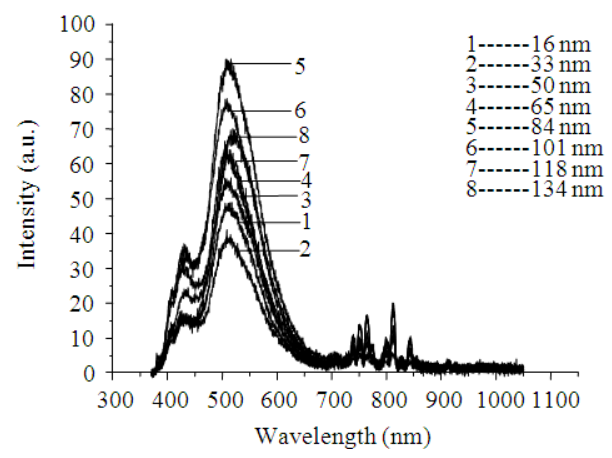

Fig. 3: Photoluminescence spectrum for different thickness of $\mathrm{Alq}_{3}$ with $55 \mathrm{~nm}$ of NPB

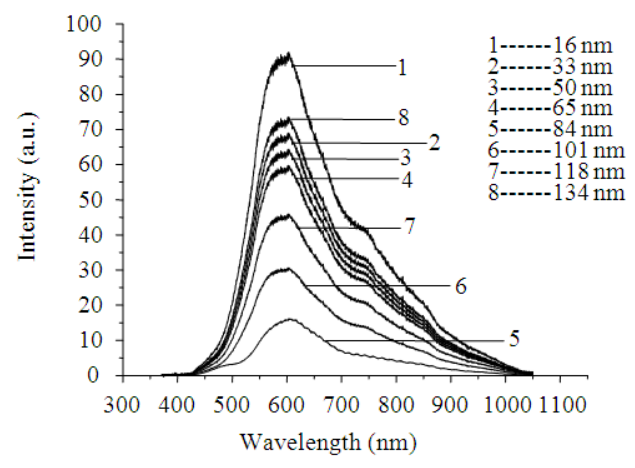

Fig. 4: The reflectance light for different thickness of $\mathrm{Alq}_{3}$ with $55 \mathrm{~nm}$ of NPB

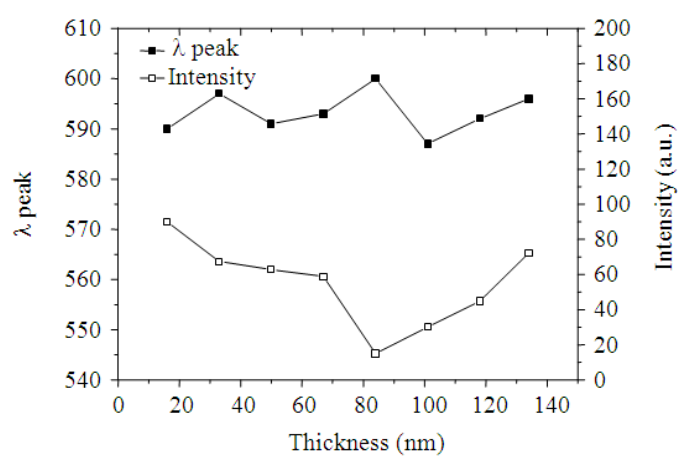

Fig. 5: $\lambda$ peak and relative intensity of reflectance of different thickness of $\mathrm{Alq}_{3}$ with $55 \mathrm{~nm}$ of NPB 


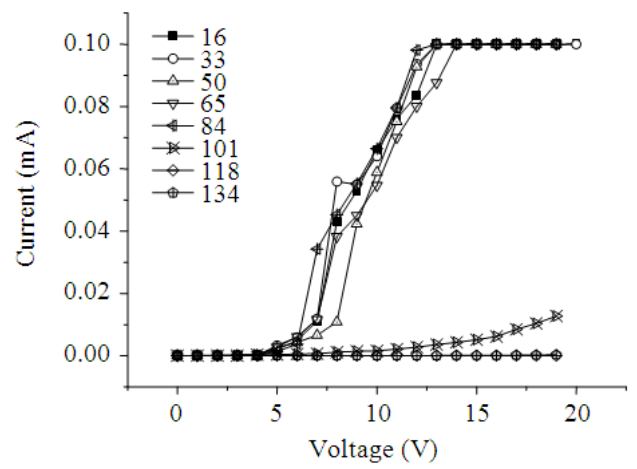

Fig. 6: Current versus voltage characteristics for devices with various thickness of $\mathrm{Alq}_{3}$

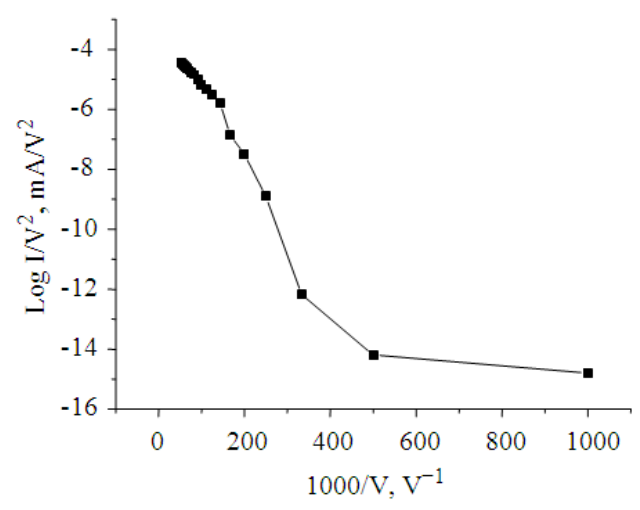

Fig. 7: The corresponding Fowler-Nordheim plot for $84 \mathrm{~nm} \mathrm{Alq}{ }_{3}$ and $77 \mathrm{~nm}$ PVK PLED device

The $55 \mathrm{~nm}$ of NPB as HTL while the $\mathrm{Alq}_{3}$ as ETL from 16 until $134 \mathrm{~nm}$. The spectra were measured after each layer deposition. The most dominant features common to all the spectra are the maximum shifting towards higher wavelengths with the increasing the thickness of $\mathrm{Alq}_{3}$ until $84 \mathrm{~nm}$. Further increase the thickness of $\mathrm{Alq}_{3}$ until $101 \mathrm{~nm}$, the maximum shifting towards lower wavelengths.

Figure 6 gives the I-V characteristics of the double layers OLEDs with various thickness of $\mathrm{Alq}_{3}$ with 55 $\mathrm{nm}$ of NPB. It can be found that the current initially decrease with the increasing of $\mathrm{Alq}_{3}$ thickness. As the thickness goes up to $84 \mathrm{~nm}$, the current turns to increase.

Figure 7 shows the Fowler-Nordheim plot for the devices indicated straight line, which implies that the current were due to field emission. Figure 8 show the turn on voltage versus the thickness of $\mathrm{Alq}_{3}$ for OLEDs devices. It can be observed that the turn on voltage initially shifts towards higher voltage as the $\mathrm{Alq}_{3}$ thickness increase from 16-65 $\mathrm{nm}$. Then, the turn on voltage drop to $5.1 \mathrm{~V}$ when the $\mathrm{Alq}_{3}$ thickness is $84 \mathrm{~nm}$.

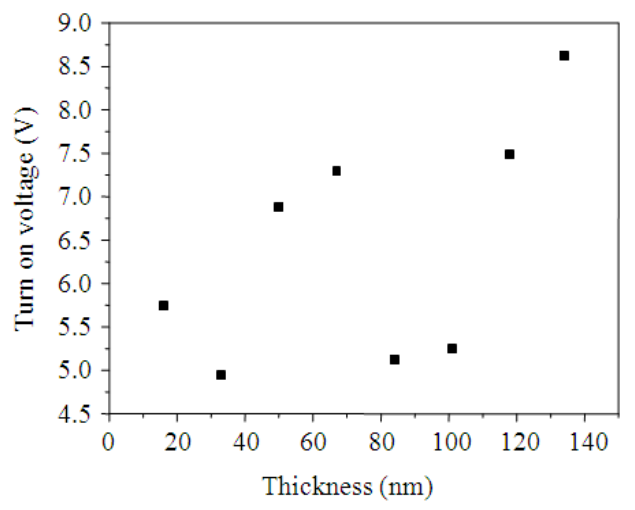

Fig. 8: Turn on voltage for OLED devices with different thickness of $\mathrm{Alq}_{3}$

\section{DISCUSSION}

It can be observed that the thickness of the $\mathrm{Alq}_{3}$ strongly effect the luminescent intensity. The luminance, initially increases from the 16 until $101 \mathrm{~nm}$ of the blocking- $\mathrm{Alq}_{3}$ layer, then drops as the thickness increases further. In terms of efficiency, the device with $84 \mathrm{~nm}$ of the blocking- $\mathrm{Alq}_{3}$ layer shows the highest luminescence intensity. The observed results can be explained in terms of the hole-blocking effect by the $\mathrm{Alq}_{3}$ layer to balance the electron and hole current. With $84 \mathrm{~nm}$ of the blocking- $\mathrm{Alq}_{3}$ layer, the balance is optimized, while a further increase in thickness causes most of the holes to be blocked at the hole transport layer. However, the intensity reflectance light increase when the thickness of $\mathrm{Alq}_{3}$ increase from 84-134 nm.

The $\mathrm{Alq}_{3}$ molecules indeed trap the electron at a low thickness layer. The current at $84 \mathrm{~nm}$ of $\mathrm{Alq}_{3}$ is higher than the other thickness. Hence, the maximum current efficiently is improved in term of the balance electron and hole current is achieved.

Further increase the thickness of $\mathrm{Alq}_{3}$, the turn on voltage increase to $8.6 \mathrm{~V}$. The increase in turn on voltage is due to the increase in trap density, where many electrons are trapped in the bulk. Thus, higher voltage is required to transfer the electrons to the interface of NPB and $\mathrm{Alq}_{3}$ and the electron enhance the injection.

Figure 9 shows a band diagram of ITO/NPB $(55 \mathrm{~nm}) / \mathrm{Alq}_{3}(84 \mathrm{~nm}) / \mathrm{Al}(300 \mathrm{~nm})$, device. The optical energy band gap of the NPB was $2.8 \mathrm{eV}$ between the HOMO energy level, $5.1 \mathrm{eV}$, measured by cyclic voltametry and the LUMO, $2.3 \mathrm{eV}$, calculated from absorption spectrum, which was consistent with the result of the optical threshold (Muller et al., 2006). 


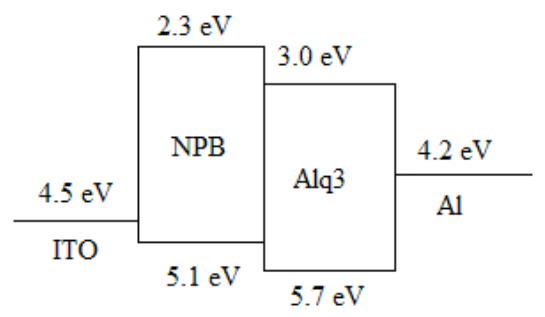

Fig. 9: Band diagram of ITO/NPB $(55 \mathrm{~nm}) / \mathrm{Alq}_{3}(84$ $\mathrm{nm}) / \mathrm{Al}(300 \mathrm{~nm})$ device

The energy diagram is essential to understand and optimize the device performance. The $\mathrm{Alq}_{3} \mathrm{HOMO}$ level is around $5.7 \mathrm{eV}$, indicating that a small energy barrier $(\sim 0.6 \mathrm{eV})$ for hole injection exists when compared with the HOMO level for NPB. The HOMO of NPB is above the HOMO of $\mathrm{Alq}_{3}$. This make holes is easily enter the ETL. In addition with the LUMO of $\mathrm{Alq}_{3}$ is below the NPB, it will enhance the electron confined in the ETL (Hung and Chen, 2002). This latter is $0.6 \mathrm{eV}$ below the ITO Fermi level. On the other hand, an electron energy barrier of about $1.2 \mathrm{eV}$ is present between the $\mathrm{Al}$ electrode Fermi level and the $\mathrm{Alq}_{3}$ LUMO level, suggesting that for the OLEDs studied in this study, holes are more efficiently injected than electrons. With the $84 \mathrm{~nm}$ of $\mathrm{Alq}_{3}$, the luminescent of the OLEDs can be enhanced to have a desirable EL color as well as high luminance efficiency.

\section{CONCLUSION}

The OLEDs with the ITO/NPB/Alq $3 / \mathrm{Al}$ has been successfully fabricated using thermal evaporation method. The characteristics of the device were carried out using fiber optics PL, I-V two-point probe and a single beam spectrophotometer. The device with $84 \mathrm{~nm}$ of $\mathrm{Alq}_{3}$ produced the lowest current turn on voltage which is $5.1 \mathrm{~V}$ and gives the highest intensity of the light emission.

\section{ACKNOWLEDGEMENT}

Financial support received from Malaysia Government through Fundamental Research Fund (FRGS 01-11-08-664FR/5523664) is gratefully acknowledged. Mei Yee is grateful for the financial support of NSF scholarship (2009-2010).

\section{REFERENCES}

Bouzid, S.B., F. Bousbih, A. Hamdouni, R. Chtourou and J.C. Harmand et al., 2005. Photocurrent and photoluminescence investigations of GaInNAs and GaInNAs(Sb) quantum wells grown by molecular beam epitaxy. Am. J. Applied Sci., 2: 1370-1374. http://www.scipub.org/fulltext/ajas/ajas291370-1374.pdf

Coe, S., W.K. Woo, M. Bawendi and V. Bulovi, 2002. Electroluminescence from single monolayers of nanocrystals in molecular organic devices. Nature, 420: 800-803. DOI: $10.1038 /$ nature 01217

Hung, L.S. and C.H. Chen, 2002. Recent progress of molecular organic electroluminescent materials and devices. Mater. Sci. Eng. R.: Rep., 39: 143-222. DOI: $10.1016 / \mathrm{S} 0927-796 \mathrm{X}(02) 00093-1$

Kanno, H., Y. Hamada and H. Takahashi, 2004. Development of OLED with high stability and luminance efficiency by co-doping methods for full color displays. IEEE J. Select. Top. Quant. Elect., 10: 30-36. DOI: 10.1109/JSTQE.2004.824076

Lim, M. Y., W.M.M. Yunus, Z.A. Talib, A. Kassim and C.F. Dee et al., 2010a. Electrical and optical properties of $\mathrm{N}, \mathrm{N}^{`}$-bis (inaphthyl)-N,N`-diphenyl1,1`-biphenyl-4,4`-diamine as hole transport layer in organic light emitting devices. Am. J. Eng. and Applied Sci., 3: 64-67. http://www.scipub.org/fulltext/ajeas/ajeas3164-67.pdf

Lim, M. Y., W.M.M. Yunus, Z.A. Talib and A. Kassim, 2010b. Optical properties for N,N'-bis (lnaphyhly)- N,N'-diphenyl-1,1'-biphenyl 4,4'diamine and tris (8-hydroxyquinolinato) aluminum in organic light emitting devices. Nat. Sci., 2: 631-634. DOI: $10.4236 / \mathrm{ns} .2010 .26078$

Muller, J., J. Nowoczin and H. Schmitt, 2006. Composition, structure and optical properties of sputtered thin films of CuInSe 2 . Thin Solid Films, 496: 364-370. DOI: 10.1016/j.tsf.2005.09.077

Yoon, Y.B., T.W. Kim, H.W. Yang, J.H. Kim and J.H. Seo et al., 2007. Organic light-emitting devices with a mixed layer acting as a hole transport and as an emitting/electron transport layer. Thin Solid Films, 515: 5095-5098. DOI: 10.1016/j.tsf.2006.10.020

Zhao, J., J.A. Bardecker, A.M. Munro, M.S. Liu and Y. Niu et al., 2006. Efficient CdSe/CdS quantum dot light-emitting diodes using a thermally polymerized hole transport layer. Nano Lett., 6: 463-467. PMID: 16522043 